\title{
Dental Fluorosis and its Indices, what's new?
}

\author{
Abiodun-SolankeIyabo M. Funmilayo ${ }^{1}$, Ajayi Deborah Mojirade ${ }^{2}$ \\ ${ }^{1,2}$ Department of Restorative Dentistry, College of Medicine/University of Ibadan. Nigeria
}

\begin{abstract}
Since the description of dental fluorosis as a disease entity by Fredrick McKay and GV Black in 1916, several indices have been used to categorize the different forms of dental fluorosis. Such indices include Dean's, Thylstrup and Fejerskov's(T-F), Total Surface Index of Fluorosis(TSIF) etc. However, none of these indices that was introduced in the $20^{\text {th }}$ century is without shortcomings or limitations, which eventually led to the discovery of newer indices with continuous scale such as the Visual Analogue scale, Quantitative Light Fluorescence and Polarized white lightimages .
\end{abstract}

Keywords: Dental, Fluorosis, Indices, Update

\section{Introduction}

Dental fluorosis, a specific disturbance in tooth formation and an esthetic condition, is defined as a chronic fluoride -induced condition in which enamel development is disrupted and the enamel is hypomineralised ${ }^{1}$. Simply put, dental fluorosis is a condition in which an excess of fluoride is incorporated in the developing tooth enamel. Fluorosis has a characteristic appearance and distribution in the mouth ${ }^{1-5}$. The severity of dental fluorosis depends on when and for how long the over exposure to fluoride occurs, the individual response, weight, degree of physical activity, nutritional factors and bone growth ${ }^{6}$. However, the most important risk factor for fluorosis is the total amount of fluoride consumed from all sources during the critical period of tooth development ${ }^{6}$.

In 1906, in North America Dr. Fredrick McKay first observed a discoloration called "Colorado Brown Stain" "7 which led to the discovery of fluoride's caries preventive action. Dr. GV Black later got involved in 1909 and by 1916 Mckay and Black conducted a study and hypothesized that an unidentifiable factor in drinking water was responsible for the enamel mottling, and in 1931, HV Churchill identified fluoride as the causative agent. Dean conducted a survey in 1931 and develop an index for fluorosis in 1934 known as Dean's index

Clinically, mild enamel fluorosis is seen as diffuse white spots or white opaque lines or striations or a white parchment -like appearance of the tooth surface that run horizontally across the enamel. These may be invisible to the individuals and clinicians but often can be seen after the enamel has been dried. The opacities may coalesce to form white patches. In the moderate or more severe forms, the enamel may become discolored and /or pitted ${ }^{8-11}$ due to uptake of extrinsic stains mainly from the diet. At high concentrations of fluoride, discrete or confluent pitting of the enamel surface is seen, accompanied by extrinsic stain ${ }^{9-11}$.

Fluorosis is symmetrically distributed, but the severity varies among the different types of teeth ${ }^{12,13}$. Teeth that develop and mineralize later in life such as premolars have a higher prevalence of fluorosis, and are more severely affected ${ }^{13,14}$. Rarely are the primary dentition and lower incisors affected.

Several indices have been used to measure this diseased condition, among which are Dean's index, ThylstrupFejerskov index etc It is expected that an index should be measurable, sensitive, and reliable

\section{Measurement of Dental Fluorosis in the $20^{\text {th }}$ Century}

The instrument employed in dental fluorosis measurement are indices and imaging techniques. It is necessary to measure dental fluorosis for surveillance purposes, research purposes and for treatment decisions. An index for measuring any condition should be sensitive, easy to understand and reliable.

In the $20^{\text {th }}$ century, the various indices used for measurement of dental fluorosis are;

1. Dean's Index- 1934

2. Community Fluorosis Index-1946

3. Thylstrup-Fejerskov Index-1978

4. Tooth surface Index of Fluorosis -1984

5. Fluorosis Risk Index-1990

6. The developmental defects of enamel index-1982

11.1 Dean's Index ${ }^{15}$

It was first described in 1934 and was later modified in 1942. The index was developed to gain an understanding of the relationship between fluoride concentrations in drinking waters and mottled enamel. It was 
designed to reflect the clinically visible features of dental fluorosis in a population and approximate the actual biologic effects of fluoride on developing dental enamelIt emphasizes the aesthetic aspect of dental fluorosis.It became the most universally acceptable classification system for dental fluorosis found on two or more teeth. If two teeth are not equally affected, the less affected will be scored. This index categorizes dental fluorosis on a six point ordinal scale as normal, questionable, very mild, mild, moderate, and severe as shown below.

Normal : the enamel represents the usual transluscent semi vitriform type of structure, surface is smooth, glassy, pale, creamy white transluscent.

Questionable - The enamel discloses slight aberrations from the translucency of normal enamel ranging from a few white flecks or occasionalwhite spots.

Very mild:small opaque paper white area scattered irregularly over the tooth covering less than $25 \%$ of tooth surface. Bicuspids / second molars not showing more than 1-2mm of white opacity at the tip of summit of cusps are also frequently involved in this classification.

Mild : opaque white area in the enamel of the tooth covering less than $50 \%$ of the tooth surface.

Moderate :All enamel tooth surfaces are affected, and surfaces subject to attrition show marked wear. Brown stain may be present.

Severe :. All enamel surfaces are affected and hypoplastic brown stains are widespread and teeth often present as corroded appearance. The major diagnostic sign of this classification is the discrete or confluent pitting.

Dean's index has remained popular because of its simplicity and its ability to make comparisons with numerous earlier studies. In their studies of the assessment of examiner reliability of this index, Kumar et $\mathrm{al}^{16}$ showed good to excellent agreement beyond chance in the use of the index. Agreement on the presence or absence of fluorosis using Dean's definition of fluorosis ranged from $92-97 \%$.

Although Dean's index has been in use for over 50 years, it is not without shortcomings. Dean's classification "questionable" has caused a lot of confusion, though, Dean intended the questionable category to be used in cases where the examiner was unsure as to whether opacities should be defined as normal/ very mild. It is also considered that DI is not able to give sufficient information on the distribution of dental fluorosis within the dentition especially with its lowest score- the questionable score. DI scores are ordinal and thus creating problems in data analysis and it is argued that DI scores are not sensitive enough. The index cannot discriminate between severe forms of dental fluorosis. Consequent to these limitations, other scoring systems have been developed.

\subsection{The Community Fluorosis Index-1946}

This was also proposed by Dean. This index awards weights to the different scores in the Dean's index. Normal is awarded $0,0.5$ to questionable and $1,2,3,4$ to very mild, mild, moderate and severe respectively

\subsection{Thylstrup and Fejerskov Index- (TFI)}

This TFI was proposed by Thylstrup and Fejerskov $(1978)^{17}$ with the aim of overcoming the shortcomings of the Dean's index. Like the DI, the TFI is a tooth based scoring system that produces a maximum of 28 scores per subject. It is a 10 point classification scale with numeric values from $0-9$. This original index (with 10 categories involving description of all tooth surfaces)of fluorosis attempts to correlate clinical appearance with pathological changes in tissue. It therefore is a useful tool when evaluating dental fluorosis severity in epidemiological studies. However, it uses ordinal scale and therefore the scores should be considered only arbitrary points along a continuum of change. The index was later modified to be based solely on examination of facial tooth surfaces ${ }^{18}$

Score Criteria

0 Normal translucency of enamel remains after wiping and drying of the surface

1 Narrow opaque/white lines running across the tooth surface. Slight snow capping of cusps or incisal edges may also be seen.

2 Smooth surfaces. More pronounced lines of opacity that follow the perikymata. Occassionally confluence of adjacent lines.

Occlusal surfaces: Scattered areas of opacity less than $2 \mathrm{~mm}$ in diameter and pronounced opacity of cuspal ridges. Snow-capping is common.

3 Smooth surfaces: Merging and irregular cloudy areas of opacity. Accentuated drawing of perikymata often visible between opacities.

Occlusal surfaces: Confluent areas of marked opacity. Worn areas appear almost normal but usually circumscribed by a rim of opaque enamel

4 Smooth surfaces: The entire surface exhibits marked opacity or appears chalky

white. Parts of surface exposed to attrition appear less affected.

Occlusal surfaces: Entire surface exhibits marked opacity. Attrition is often pronounced shortly after eruption. 
5 Smooth surfaces and occlusal surfaces: Entire surface displays marked opacity

Focal loss of outermost enamel(pits).less than $2 \mathrm{~mm}$ in diameter.

6 Smooth surfaces: Pits are regularly arranged in horizontal bands less than $2 \mathrm{~mm}$ in vertical height

Occlusal surfaces: confluent areas less than $2 \mathrm{~mm}$ in diameter exhibit loss of enamelMarked attrition.

7 Smooth surfaces: Loss of outermost enamel in irregular areas involving less than halfof the entire surface.

Occlusal surfaces: changes in the morphology caused by merging pits and marked attriton

8 Smooth and occlusal surfaces: Loss of outermost enamel involving more than half of surface

9 Smooth and occlusal surfaces: Loss of main part of enamel with change in anatomic appearance of surface.

Cervical rim of almost unaffected enamel is often noted.

The sensitivity of TFI comes from its 9 stages reflecting the histopathology and fluoride content in the enamel. It is sensitive, easy to understand, reliable and at the most outstanding for evaluating the severity of fluorosis ${ }^{19}$. TFI had an excellent reproducibility despite its extended scale, was suitable to categorize mild forms of dental fluorosis withease, due to drying of teeth before scoring and had clear description and discrimination of the categories in the lower end of the index, whereas DI lacked accuracy to discriminate within the low fluoride scores. TFI also facilitated discrimination of severe cases of dental fluorosis that were categorized in one score by DI.

\subsection{Total Surface Index of Fluorosis (TSIF)}

This was proposed by Horowitz et al $(1984)^{20}$, in an attempt to reduce some of the shortcomings of Dean's index. It allows for separate assessment of cosmetic fluorosis i.e fluorosis discoloration, staining or pitting on surfaces visible to others. According to the authors, a separate score is given to each unrestored tooth surface. Two scores are assigned to anterior teeth (from the labial and lingual aspects) and three to the posterior teeth(from the buccal, lingual and occlusal aspects). The TSIF permits a distinction between pitting and more advanced pitting and between staining alone and staining in conjunction with pittingIt was developed and used by researchers in the National Institute of Dental Research in USA.More sensitive than Dean's Index for mildest forms of fluorosis. The tooth surface index of fluorosis has identified seven types.

The TSIFScale

\section{SCORE DESCRIPTIVE CRITERIA}

Enamel shows no evidence of fluorosis

Enamel shows definite evidence of fluorosis namely areas with parchment white color that total less than one third of visible enamel. This categories include fluorosis confined only to incisal edges of anterior teeth and cusp tips of posterior teeth( snowcapping)

Parchment - white fluorosis at least one third of the visible surface, but less than two thirds.

Parchment- white fluorosis total at least two-thirds of the visible surface.

Enamel shows staining in conjunction with any of the preceding levels of fluorosis, staining is defined as an area of definitive discoloration that may range from light to very dark brown

Discrete pitting of the enamel exists, unaccompanied by evidence of staining of intact enamel. The pitted area is usually stained or differs in color from the surrounding enamel.

Both discrete pitting and staining of the intact enamel exist.

Confluent pitting of the enamel surface exists. Large areas of enamel may be missing and the anatomy of the tooth may be altered. Dark brown stain is usually present.

In comparing the 3 main indices (DI, TFI, TSIF), Pereira and Moreira concluded that if the same measuring methods are used, the three fluorosis indices had similar prevalence, however they suggested appropriate indications for each as follows:

1). Dean's index : for comparative studies between prevalence and those found in the decades of the 30's and 40's.

2). T-F Index: for clinical studies or analytical epidemiological studies.

3). TSIF: for studies in which an esthetic basis is desired for defining case and also it may be used where risk factors are identified or when the teeth may not be cleaned or dried.

\subsection{Fluorosis Risk Index}

This was proposed by Pendrys $1990^{22}$ and can been used in Analytical Epidemiological studies. It was designed to permit a more accurate identification of risk factors of enamel fluorosis and developed to allow for the identification of the time during tooth maturation at which exposure was most likely to have been experienced. More accurate identification of age specific exposures to fluoride sources and the development of enamel fluorosis. This index divides the enamel surfaces of teeth in the secondary dentition into 2 groups of surface zones

i.) Classification 1-Enamel surface zones that begin formation during the first year of life

ii.) Classification 11--- Enamel surface zones that begin formation between the third to sixth year of life 
The areas assigned to classification 1 are the incisal edges of the mandibular central and lateral incisor and maxillary central incisors, and the occlusal tables of mandibular and maxillary first molars. These surfaces are at risk of fluorosis if fluoride exposure occurs during the specified time.

For classification 11, the areas include the cervical $3^{\text {rd }}$ of the incisors, middle $3^{\text {rd }}$ of the canines and the occlusal table, incisal $3^{\text {rd }}$ and middle $3^{\text {rd }}$ of the bicuspids and $2^{\text {nd }}$ molars in both maxillary and mandibular arches.

FRI Scoring Criteria

Each visible surface is scored according to the following criteria.

Negative Finding

Score 1 --- No indication of fluorosis is present. Complete absence of white spots/

striations with normal tooth surface colouration.

Questionable Findings

Score' 1: Any surface zone that is questionable as to whether there is fluorosis(i.e

white spots, striations or fluorosis defects cover over $50 \%$ or less of the

surface zone) should be scored as 1 .

Score 7 : Any surface that has an opacity that appears to be a non- fluorotic

opacity should be scored 7

Positive Findings

Score 2: Positive for mild-moderate fluorosis, greater than $50 \%$ of the zone

displays parchment white striations typical of enamel fluorosis

Incisal edges and occlusal tables will be scored as positive for enamel fluorosis if greater than $50 \%$ of that surface is marked by snow capping typical of enamel fluorosis.

Score 3 :Positive for severe fluorosis. A surface will be diagnosed as positive for severefluorosis if greater than $50 \%$ of the zone displays pitting, staining and deformity.

Surface Zone Excluded

Score :=9

A surface zone is categorized as excluded when any of the following condition exists:

1. Incomplete eruption

2. Presence of orthodontic appliance and bands

3. Surfaces crowned / restored

4. Presence of gross plaque / debris

The FRI scoring system appears to be directed at risk assessment based on prevalence of fluorosis rather than severity within subjects. A direct comparison of the four index systems for a common population would be highly desirable for better understanding of relationships among these scoring systems.

\subsection{Developmental defects of enamel}

This was proposed in $1982^{23}$ by Commission on Oral Health, Research and Epidemiology arising from a lack of a well-defined and internationally accepted classification of enamel defects. The index was designed to promote use of standard terminology, simplicity and to provide an effective system for recording enamel defects in large studies. The first version was, however complicated and difficult to analyze thus, Clarkson and O'Mullane ${ }^{24}$ suggested a modified and simplified version of the index that has now been widely adopted (Federation Dentaire Internationale, ${ }^{25}$ )

Modified DDE Index for use in screening studies (Clarkson and O'Mullane, 1989)

\section{Type of Defect}

Normal

Demarcated Opacities

Diffuse Opacities

Hypoplasia

Other defects

Demarcated \& diffuse Opacities combined

Demarcated Opacities plus hypoplasia

Diffuse Opacities plus hypoplasia
Code

0

3

4

5

6

7Demarcated \& Diffuse Opacities plus hypoplasia 8

DDE is now the most currently and widely used index in the United Kingdom to study enamel defects $^{26}$. DDE index is descriptive but not specific for fluorosis thus when the objective of the study is to find the prevalence/ severity of fluorosis, DDE index might not be suitable. However, Sabieha and Rock ${ }^{27}$ found a good correlation between the index and TF- index and concluded that either will yield broadly comparable results where it may be expected that fluoride induced opacities will form a significant proportion of all enamel defects found. 


\section{New Concept in measurement of Dental Fluorosis}

The use of clinical photographs in measurement of dental fluorosis came into limelight in the $21^{\text {st }}$ century.Photographs came into use because the indices that wereused until then were subjective and prone to bias. Clinical photograph can be taken during examinations and graded remotely. Photography also enables archiving, assessment of longitudinal changes, scoring by multiple examiners, remote examiner scoring is enabled, and enhances production of training sets for examiner calibration.

111.1 Measurement of Dental Fluorosis: Prospects

a) A visual analogue scale(VAS) by Vieira et al 2005.

b)Quantitative Light Fluorescence Assessment of Dental Fluorosis by Pretty et al 2006.

c) Quantitative Light Fluorescence and Polarised white light assessment of dental fluorosis—Pretty et al 2012.

\subsubsection{A Visual Analogue Scale (VAS)}

This scale was developed by Vieira at alin $2005^{28}$. It was developed because previous indices were not on a continuous scale. It adapts a $100 \mathrm{~mm}$ continuous scale originally developed for pain measurement. It is also use to measure alertness after sleep, attitude toward the environment, quality of life and anxiety ${ }^{29}$. The main advantages of the VAS for dental fluorosis over the ordinal scales for dental fluorosis( such as TFI,DI,TSIF) are the continuity of the scale, its simplicity and its precision.It uses visual indicators to measure dental fluorosis. These indicators guide examiners through the scale and help them use it precisely.

\subsubsection{Quantitative Light Fluorescence (QLF)}

This was described by Pretty et al in $2006^{30}$. This scale was developed as a result of some pitfalls identified with traditional photography such as the inability toassess dental fluorosis against other enamel defects,. The phenomenon of personal thresholding particularly at low levels of fluorosis severity with differences in the application of diagnostic criteria ${ }^{31,32}$ and the variability in inter and intra-examiner agreement are also issues of concern with traditional photography. Training of examiners will also be costly and complex. Therefore,the need for assessment of dental fluorosis by an automated grading system. . The principle used in QLF is to compare changes in fluorescence between 'sound and 'unsound' enamel. Images are usually assessed by a computer software. However, specular reflection is a cofounder. An inherent limitation of QLF is the inability to differentiate florescence loss as a result of fluorosis; other forms of developmental defects and tooth surface phenomena such as enamel fractures and extrinsic $\operatorname{stain}^{33,34}$.

\subsubsection{Polarized White Light Images}

These remove the problem of specular reflection. Pretty et al, $2012^{35}$ proposed a new system that combined fluorescent imaging with polarized white light. Better images were obtained for assessment by the automated grading system.

\section{Conclusion}

Dental fluorosis used to be regarded as merely a cosmetic problem but now, it is seen as a condition that affects the esthetic, emotional, social, and even psychological aspects of an individual's life. Therefore, research is ongoing to improve the validity of its instruments of measure.

\section{References}

[1]. O Ferjerskov, F ManjiandVBaelum,. The nature and mechanism of dental fluorosis in man, Journal of Dental Research ,69( Special issue), 1990, 670-692.

[2]. HT Dean, FA Arnold, and E Elvove , Domestic water and dental caries V. Additional studies of the relation of fluoride domestic water to dental caries experience in 4,425 white children aged 12-14 years in 13 cities in 4 states, Public Health Report, 1557, ,1155-1179.

[3]. A Thyistrup, and O Fejerskov, Clinical appearance of dental fluorosis in permanent teeth in relation to histological changes, Community Dentistry and Oral Epidemiology,6, 1978,315-328.

[4]. O Fejerskov, J Kragstrup ,and A Richards, Fluorosis of bone and teeth, in: J Ekstrand, O Fejerskov , IM Silverston (Ed), Fluoride in dentistry, (Copenhagen : Munksgaard 1989) 190-228.

[5]. O Fejerskov, F Manji, V Baelum,, and IJ Moller,Indices for measuring dental fluorosis, in: O Fejerskov, F Manji,,V Baelum,, and D Muller D (Ed) Dental Fluorosis- a handbook for health workers, (Copenhagen: Munksguard 1988) 44-50.

[6]. J Clarkson, Review of terminology, classifications, and indices of developmental defects of enamel. Advances in Dental Research,3(2), 1989,104-109.

[7]. FS Mckay, and GV Black, An investigation of motted teeth: an endemic developmental imperfection of the enamel of teeth heretofore unknown on the dental literature of dentistry, Dental Cosmos 58, 1916, 477-484.

[8[. $\quad$ AK Mascarenhas, Risk factors for dental fluorosis:a review of recent literature,Peadiatric Dentistry,22(4),2000,269-277.

[9]. O Fejerskov et al, Clinical and structural features and possible pathogenic mechanisms of dental fluorosis, Scandinavian Journal of Dental Research 85(7),1977, 510-534.

[10]. O Fejerskov, V Baelum, and A Richards, Dose-Response and dental fluorosis, in O Fejerskov, J Estrand and BA Burt (Ed), (Copenhagen ,Munksgaard 1996)156-166. 
[11]. B Sundstrom, and H Myhrberg, Light and scanning electron microscopy of fluorosed enamel from human permanent teeth, Caries Research 12(6), 1978, 320-329

[12]. B Sundstrom, WL Jongebloed, · M Bouchouchi, Morphology of outer regions of fluorosed deciduous enamel. Caries Research 14(6), 1980, 381-388.

[13]. MJ Larsen, A Richards, and O Fejerskov, Development of dental fluorosis according to age at start of fluoride administration, Caries Research 1, 1985, 19-27.

[14]. EG Rozier,.Epidemiological indices for measuring the clinical manifestations of dental fluorosis: Overview and Critique, Advances in Dental Research, 8(1),1994, 139-155.

[15]. HT Dean, Classification of mottled enamel diagnosis, Journal of American Dental Association, 21,1934, 1421

[16]. JV Kumar, PA Swangu, PNOpima, and EL Green, Dean's Fluorosis index: An assessment of examiners reliability,Journal of Public Health Dentistry 60(1), 2000, 57-59.

[17]. A Thystrup,OFejerskov, Clinical appearance of dental fluorosis in permanent teeth in relation to histological changes, Community Dentistry and Oral Epidemiology,6(6), 1978, 315-328.

[18]. OFejerskov, F Manji, V Baelum, and IJ Muller, Dental fluorosis- a handbook for health workers. (Copenhagen,Munksgaarrd $1^{\text {st }}$ edition 1966)

[19]. C Fraysse, JA Pouezat, Relevance of epidemiological indices for assessing dental fluorosis, (World Health Statistics Quarterly), Rapport Trimestriel de statistique 47(2), 1994, :62-64.

[20]. HS Horowitz, WS Driscoll, RJ Meyers, SB Heifetz, and A Kingman, A new method for assessing the prevalence of dental fluorosis: The Tooth Surface Index of Fluorosis, Journal of American Dental Association 109, 1984, 37-41.

[21]. AC Pereira, BW Moreira, Analysis of three dental fluorosis indexes used in Epidemiological Trials, Brazilian Dental Journal, 10(1), 1999, 29-37.

[22]. DG Pendrys, The fluorosis risk index: a method for investigating risk factors, Journal of Public Health Dentistry, 50, 1990, :291298.

[23]. JAinamo,, TN Cuttress, An epidemiological index of developmental defect of enamel (DDE),. International Dental Journal32, 1982,159-167.

[24]. J Clarkson, D O'Mullane, A modified DDE index for use in epidemiological studies of enamel defects, Journal of Dental Research, 68, 1989, 445-450.

[25]. FederationDentaireInternationale, A review of developmental defects of enamel index (DDE). Commission on Oral Health Research and Epidemiology, International Dental Journal, 42, 1992,411-426.

[26]. PJHolloway,,RP Ellwood, The prevalence causes and cosmetic importance of dental fluorosis in the UK: a review, Community Dental Health, 14,1997, 148-155.

[27]. AM Sabieha, WP Rock, A comparison of clinical and photographic scoring using the TF and modified DDE indices, Community Dental Health, 15(2),1998, 82-87.

[28]. APGF Vieira,, HP Lawrence HP, H Limeback,, FC Sampaio,, and M Grynpas,. A visual analogue scale for measuring dental fluorosis severity, Journal of American Dental Association, 135(7), 2005, 895-901.

[29]. M Briggs,,JSCloss, A descriptive study of the use of visual analogue scales and verbal rating scales for the assessment of postoperative pain in orthopedic patients, Journal of Pain Symptom and Management,18(6), 1999,438-446.

[30]. IA Pretty, JA Tavener,, D Browne,DS Brettle,H Whelton,, and RP Ellwood, Quantification of dental fluorosis using fluorescence imaging,Caries Research, 40(5), 2006, 426-434.

[31]. R Ellwood, D O'Mullane, J Clarkson, W Driscoll,A comparison of information recorded using the ThystruppFejerskov index, Tooth Surface Index of Fluorosis and Developmental Defects of Enamel index, International. Dental Journal, 44(6), 1994, 628636.

[32]. JTravener, RM Davies, RP Ellwood. Agreement amongst examiners assessing dental fluorosis from digital photographs using the TF index, Community Dental Health, 24(1), 2007, 21-25.

[33]. MG McGrady, RP Ellwood, A Taylor, A Maguire, M Goodwin M et al, Evaluating the use of Fluorescent Imaging for the Quantification of Dental Fluorosis, BMC Oral Health 12(47), 2012, 366. doi: 10.1186/1472-6831-12-47.

[34]. BIAngmar-Månsson, J ten Bosch, Quantitative light-induced fluorescence (QLF): a method for assessment of incipient caries lesions, Caries Research, 40(5), 2006, 426-434.

[35]. IA Pretty IA et al. Quantitative Light fluorescence (QLF) and Polarized White Light (PWL) assessments of dental fluorosis in an epidemiological setting, BMC Public Health 12(1), 2012, 366. doi:10.1186/1471-2458-12-366. 